\title{
Study on an Algorithm for Generating the Multiple Watermark Information using 2D Barcode
}

\author{
Jizhe $\mathrm{Cui}^{1}$ and Jongweon $\mathrm{Kim}^{2}$ \\ ${ }^{1}$ Dept. of Information Management \& Systems, Yanbian University, China \\ ${ }^{2}$ Dept. of Intellectual Property, Sangmyung University, Korea \\ ${ }^{1}$ cuijizhe@gmail.com, ${ }^{2}$ jwkim@smu.ac.kr (Corresponding Author)
}

\begin{abstract}
In this paper, we propose a multiple watermarking algorithm which embeds an integrated watermark into content using traditional watermarking algorithm. The integrated watermark consists of multiple watermark information and it is useful to trace illegal distribution. The information is generated by using Dot Code A, which can express multiple user information with error correction. An orthogonal transform and code division multiplexing technology are used to spread Dot Code A. This also ensures the algorithm is robust. The embedding range of the $A C$ coefficients is within [-0.5, -0.3] and [0.3, 0.5] in the discrete cosine transform domain. At an embedding strength of 0.7, the signal-to-noise ratio of the test audio is over $53 \mathrm{~dB}$, and we are able to extract the Dot Code A from the watermarked audio exactly.
\end{abstract}

Keywords: Digital Watermark, Multiple Watermark, Dot Code A, Illegal Distribution

\section{Introduction}

Recently, digital watermarking algorithms for the purpose of copyright protection have been actively developed. Especially, multiple watermarking algorithms that enable the confirmation of copyright at the content value chain [1-2]. The multiple watermarking algorithm has been discussed by M. Bami [3]. In general, traditional multiple watermarking algorithms can be divided into two categories. In the first, the content is divided into a number of blocks, and a different watermark is embedded into each block [4-5]. Agarwal's watermarking algorithm using SVD(singular value decomposition) is a kind of this type [6]. Agarwal divided the image into RGB space, extracted the orthogonal matrices using SVD, and embedded the watermark into SVD domain. The second method is to integrate multiple sets of information into the content using a single watermarking algorithm [5].

The former algorithm has several problems. It is vulnerable to various attacks, and is not particularly efficient for information hiding because it attempts to embed too much information, which is spread across different blocks. The latter algorithm requires a large amount of raw data, such as mapping tables, because it must pre-process the embedding information [1-2]. For solving the problems, several researches using PDF417 and QR (quick response) code as the watermark have been performed [7-8].

In this paper, we proposed an audio watermark algorithm to solve these problems. We develop a coding method for watermark generation using a 2D barcode, Dot Code A, and code division multiplexing. Our algorithm fully utilizes the error correction capability of 2D barcodes, and introduces an orthogonal code feature and chip sequence principle to improve its robustness. 
In Section 2, we describe Dot Code $\mathrm{A}$ and the orthogonal transform (spread spectrum), and Section 3 introduces the principle of multiple watermarking and presents the embedding and extraction processes. Section 4 evaluates the performance of the algorithm, and we present some conclusions in Section 5.

\section{2D Code and Orthogonal Transform}

2D code has several advantages such as high payload, space availability, and error correction function. In order to express the multiple watermark information by $2 \mathrm{D}$ code, the proposed algorithm should define the precondition and it is as follows;

Condition 1: the watermark should contain proper amount of the information against to the attacks and multiple embedding should be possible.

Condition 2: the embedding information should not be constrained by blocks.

Condition 3: the multiple watermark information should be robust to the malicious attacks.

There are two representative codes which are multi-layer code and matrix code. The multilayer code is a cumulative and complicated code of the same type symbol because the code reader has to analyze the synch code for several layers. Because of this reason, the proposed algorithm uses the matrix code. Compared to standard 2D barcodes, such as a QR code or Data Matrix, Dot Code A has two advantages. It has four black dots, one in each corner, to represent the boundary of the barcode, and is similar in appearance to a QR code, which has three triple-layered squares in its corners. In addition, the duplicated pattern of the Data Matrix can be implemented by repeated use of Dot Code A.

Figure 1 shows some examples of Dot Code A that express 20 bits of information [9].

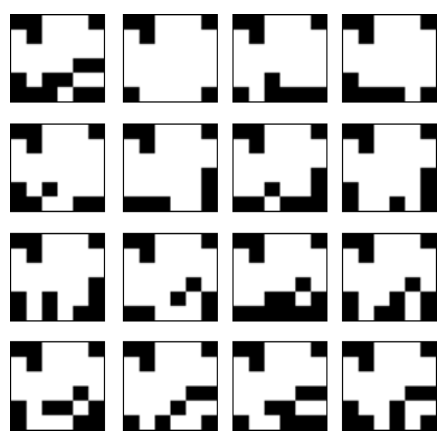

Figure 1. Dot Code A expressed 0-15

Dot Code A has the following features:

Feature 1: Data is represented by a square array of dots, from $6 \times 6$ to $12 \times 12$.

Feature 2: Black dots represent 1, and white represents 0. Four dots are printed at each corner to denote the boundary of the code.

Feature 3: The direction of the code is determined by the upper-left corner symbol. This symbol also distinguishes the symmetrical pattern.

These features are expressed by 10 dots, which make the finder pattern [9]. By modifying Dot Code A, these features are appropriate for the application of a multiple audio watermarking algorithm. 
We use the spread spectrum technique to generate the watermark from Dot Code A. If a user code sequence is $\mathrm{S}$ and another user code is $\mathrm{T}$, then the inner product of $\mathrm{S}$ and $\mathrm{T}$ is 0 , whereas the inner product of $\mathrm{S}$ and $\mathrm{S}$ is 1 . Formally:

$$
\begin{aligned}
& S \cdot T \equiv \frac{1}{m} \sum_{i=1}^{m} S_{i} T_{i}=0 \\
& S \cdot S \equiv \frac{1}{m} \sum_{i=1}^{m} S_{i} S_{i}=\frac{1}{m} \sum_{i=1}^{m} S_{i}^{2}=1 .
\end{aligned}
$$

The sequence makes Dot Code A into an orthogonal random sequence. If the code sequences are orthogonal, the orthogonal watermark information is assigned to each user and one watermark can ensure

\section{Multiple Watermarking Algorithm}

\subsection{Generation of Watermark Information}

First, the Dot Code A including user information should be changed from a uni-polar to a bi-polar sequence using the Hadamard transform. We define a Hadamard matrix $H(n, m)$ of order $n$ and column order $m$ such that:

$$
H H^{T}=n I_{n},
$$

where $\boldsymbol{I}_{n}$ is the $n \times n$ identity matrix and $\boldsymbol{H}^{T}$ is the transpose of $\boldsymbol{H}$. Consequently, the determinant of $\boldsymbol{H}$ equals $\pm n^{n / 2}$. The partitioned matrix:

$$
\left[\begin{array}{rr}
H & H \\
H & -H
\end{array}\right]
$$

is then a Hadamard matrix of order $2 n$.

For example, an $8 \times 8$ matrix can assign the user codes $H(8,1), H(8,2), H(8,3), H(8,4)$, $H(8,5), H(8,6), H(8,7)$, and $H(8,8)$. Fig. 2 shows the spread Hadamard matrices by each user information when the watermark information is " 0000 ". The Hadamard matrix is exclusively ORed by a random sequence to make a spread spectrum sequence. The random sequence is a computer-generated pseudo random number (PRN) code. The PRN sequence, which is orthogonal, spreads the Hadamard matrix. 


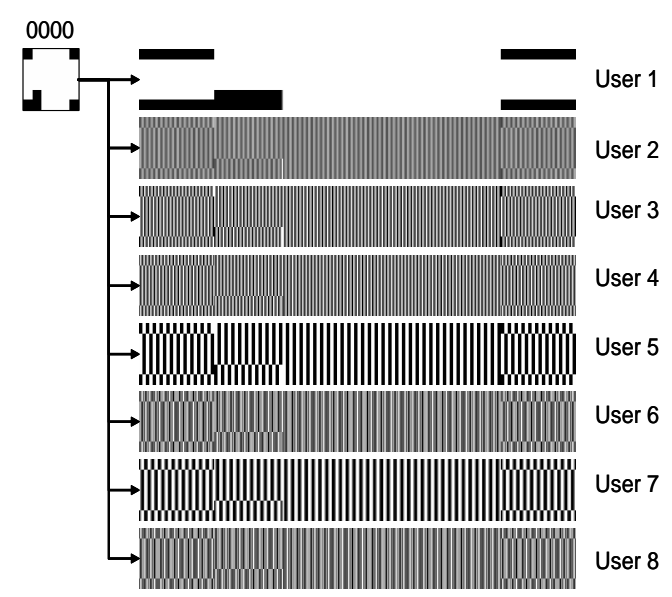

Figure 2. Watermark Generation by Spreading of User Information

The multiple watermarks are generated by spreading the Dot Code A of each user. Figure 3 shows the multiple watermark example using $\mathrm{H}(8,8)$.

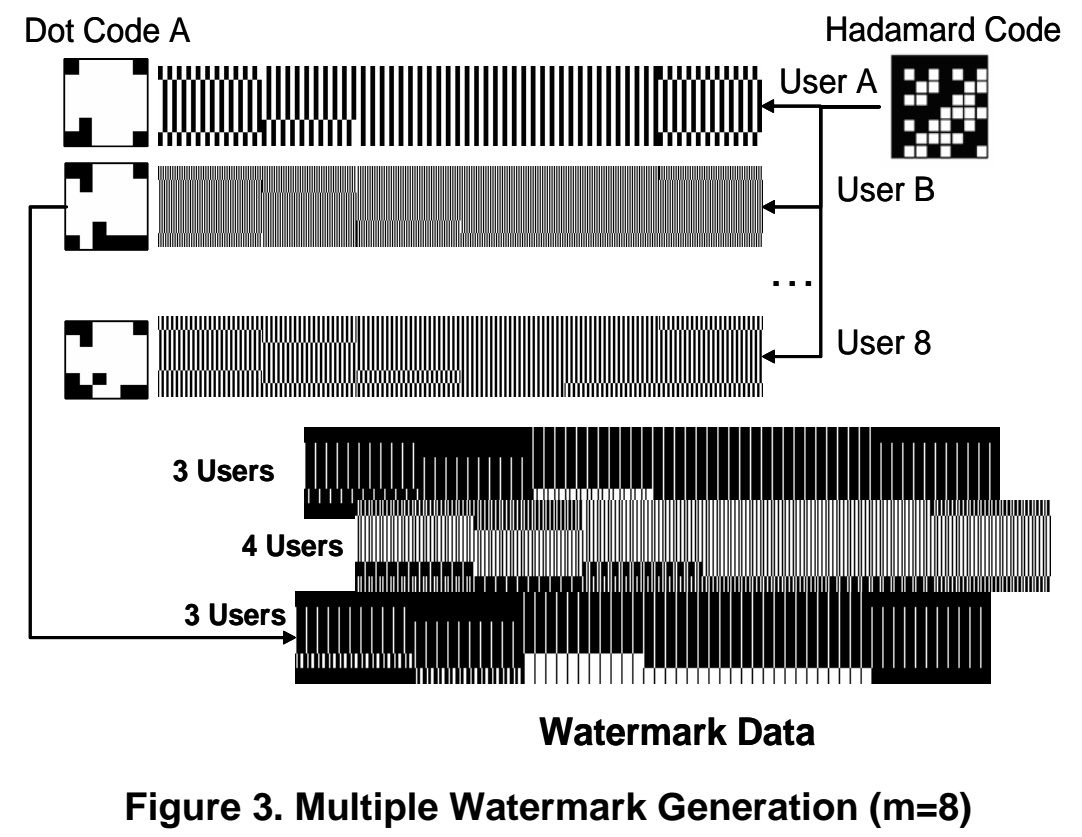

Conventional watermarking algorithms can embed multiple watermarks using space or frequency division. Generally, if an algorithm divides the space to embed the watermark, its robustness and payload cannot be ensured, because there is a degree of trade-off between these factors. In the proposed algorithm, we generate multiple watermark symbols using the orthogonality of the Hadamard transform.

If we want to embed several users' information, each user receives embedding information (Dot Code A) and a Hadamard code. The user information is spread using the code, and then the multiple watermark is generated by superposing the spread Hadamard codes. This superposition is embedded into the cover content using traditional watermarking algorithms. 


\subsection{Watermarking Algorithm}

The watermark information, in the form of the spread matrix, is embedded into the audio signal according to:

$$
Y_{i}^{\prime}=Y_{i}\left(1+\alpha x_{i}\right)
$$

where $Y_{i}$ is a DCT coefficient, $\alpha$ is the embedding strength, and $Y_{i}^{\prime}$ is the watermarked signal in the DCT domain. The embedding process is shown in Fig. 4.

In this process, the original audio is transformed into the frequency domain by DCT, and the watermark is embedded into some AC coefficients of the DCT [5]. In the proposed method, the watermark embedder selects coefficients in the range $[-0.5,-0.3]$ and $[0.3,0.5]$, and embeds the watermark accordingly. After embedding the watermark, the inverse DCT (IDCT) is computed to produce the watermarked audio.

The watermark extraction process is the reverse of the watermark embedding, as shown in Figure 5.

To extract the original audio, the extractor subtracts the original coefficients from the DCT coefficients of the watermarked audio. The watermark is extracted from the spread pattern, and then Dot Code A is extracted from the Hadamard matrix by taking the Hadamard transform. The similarity of the original code and the extracted code is given by:

$$
\sim\left(M, M^{\prime}\right)=\frac{M \cdot M^{\prime}}{\sqrt{M \cdot M^{\prime}}},
$$

where $\sim$ denotes similarity.

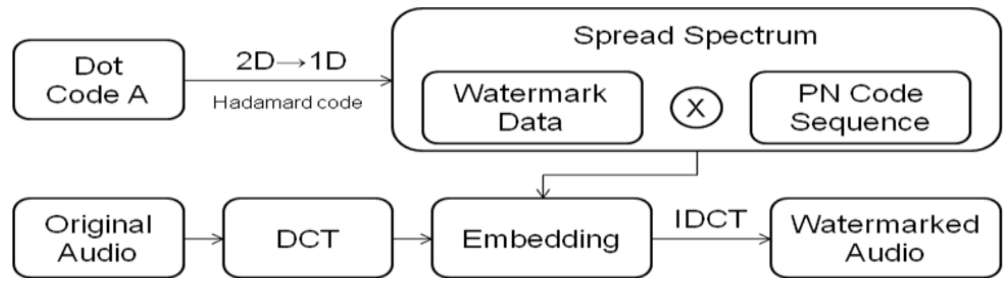

Figure 4. Embedding Process of the Proposed Algorithm

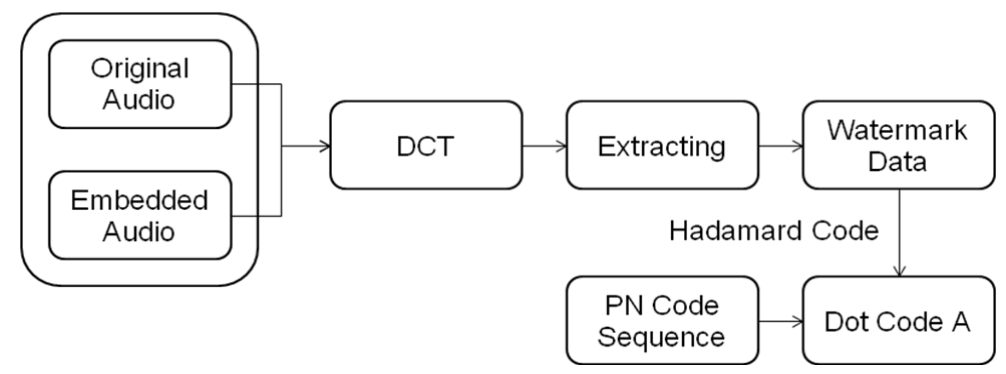

Figure 5. Extraction Process of the Proposed Algorithm

For the multiple watermarking algorithms, the two implementation methods are possible.

Method 1: In the case of multiple users, each user is assigned the user information and the Hadamard code, and then the orthogonal transform is applied using the code. The user 
information is integrated into one watermark which is embedded into content using the traditional watermarking algorithm.

Method 2: Because the proposed algorithm uses the orthogonal code each user is identified by a watermark. In this case, the user information is confirmed by each assigned code, so the amount of the information is less than the payload of Method 1.

\section{Performance Evaluation}

To evaluate the algorithm, we selected three samples of music, 'Moonlight Shadow' (Sample A), 'White Horse' (Sample B), and 'Yesterday Once More' (Sample C). The samples were recorded in stereo at 16 bits per channel and a $44.1 \mathrm{kHz}$ sampling rate. The details of the samples are shown in Table 1.

Table 1. Sample Audio Details

\begin{tabular}{|c|c|c|c|}
\hline Test sample & A & B & C \\
\hline File Format & Wav/PC & Wav/PC & Wav/PC \\
\hline Sampling size & 16 bit & 16 bit & 16 bit \\
\hline Channel & 2 & 2 & 2 \\
\hline Sampling rate & $44 \mathrm{KHz}$ & $44 \mathrm{KHz}$ & $44 \mathrm{KHz}$ \\
\hline Length(s) & 291.48 & 235.23 & 228.33 \\
\hline
\end{tabular}

After embedding the watermark, we tested the audio signal against MP3 compression (CBR-constant bit rate, 128kbps, $44.1 \mathrm{KHz}$, Stereo), echo attack and a Sub-Woofer Boost attack which is simulated by low frequency modification in the frequency domain. The echo attack was configured to have an initial volume of $70 \%$, decay of $75 \%$, and delay of $100 \mathrm{msec}$.

When the each user's Hadamard code is $8 \times 8$ and the seed of the PRN code is 1024 , the test results are shown in Table 2-Table 5. If the similarity is greater equal than 6 , the algorithm decides the watermark is detected.

Table 2. Similarity Results of Sample A

\begin{tabular}{|c|c|c|c|}
\hline Sample name & \multicolumn{3}{|c|}{ Sample A } \\
\hline Strength $(\alpha)$ & 0.3 & 0.5 & 0.7 \\
\hline No attack & 17.06 & 17.06 & 17.06 \\
\hline MP3 compression & 17.02 & 17.04 & 17.03 \\
\hline Sub WB & 6.93 & 9.20 & 11.12 \\
\hline Echo & 4.99 & 8.03 & 10.32 \\
\hline
\end{tabular}

Table 3. Similarity Results of Sample B

\begin{tabular}{|c|c|c|c|}
\hline Sample name & \multicolumn{3}{|c|}{ Sample B } \\
\hline Strength $(\alpha)$ & 0.3 & 0.5 & 0.7 \\
\hline No attack & 17.06 & 17.06 & 17.06 \\
\hline MP3 compression & 16.89 & 17.01 & 17.03 \\
\hline Sub WB & 6.82 & 9.04 & 11.03 \\
\hline Echo & 3.03 & 5.65 & 10.03 \\
\hline
\end{tabular}


Table 4. Similarity Results of Sample C

\begin{tabular}{|c|c|c|c|}
\hline Sample name & \multicolumn{3}{|c|}{ Sample C } \\
\hline Strength $(\alpha)$ & 0.3 & 0.5 & 0.7 \\
\hline No attack & 17.06 & 17.06 & 17.06 \\
\hline MP3 compression & 16.90 & 17.01 & 17.01 \\
\hline Sub WB & 6.60 & 8.97 & 11.19 \\
\hline Echo & 3.22 & 5.54 & 10.22 \\
\hline
\end{tabular}

Table 5. SNR according to Embedding Strength

\begin{tabular}{|c|c|c|c|}
\hline \multirow{2}{*}{ Sample } & \multicolumn{3}{|c|}{ SNR by Alpha } \\
\cline { 2 - 4 } & 0.3 & 0.5 & 0.7 \\
\hline A & 72.7 & 63.5 & 56.2 \\
\hline B & 71.5 & 62.1 & 54.5 \\
\hline C & 70.7 & 60.9 & 53.4 \\
\hline
\end{tabular}

The test results show that the algorithm could confirm the watermark extraction when the value of $\alpha$ was greater than or equal to 0.3 , except in the case of an echo attack, which required an $\alpha$ value of greater than or equal to 0.5 .

For embedding the multiple watermark, other algorithms are also available. When the Wushasha's algorithm [7] was applied, Dot Code A is more effective than PDF417 code. Sun's algorithm [8] shows same robustness against the compression and the extraction of the watermark information. This shows that most conventional watermarking algorithm can be used for embedding the multiple watermark which is generated by the proposed algorithm.

\section{Conclusions}

We proposed a new multiple audio watermarking algorithm using Dot Code $\mathrm{A}$ and a Hadamard transform. Our algorithm is still a non-blind form of watermarking, and so in future work we intend to improve the algorithm to allow blind watermarking. Although nonblind, the algorithm is robust against MP3 compression, Sub-Woofer Boost, and echo attacks. Indeed, the SNRs are over $50 \mathrm{~dB}$ when $\alpha=0.7$, which implies that the algorithm is highly imperceptible.

When the proposed watermark generation algorithm was applied to two traditional watermarking algorithms, the results show that Dot Code A is more effective and the possibility of the watermark detection is better at thesame condition than others.

\section{Acknowledgements}

This research project was supported by Ministry of Culture, Sports and Tourism (MCST) and from Korea Copyright Commission in 2011.

\section{References}

[1] J. Kim, N. Kim, D. Lee, S. Park and S. Lee, "Watermarking two dimensional data object identifier for authenticated distribution of digital multimedia contents", Signal Processing: Image Communication, vol. 25, no. 8, (2010), pp. 559-576.

[2] S. Dawei, "Algorithm to Embed multiple digital watermarking in BMP Image", Shanghai Communication University master course paper, (2008). 
[3] M. Bami, F. Bartolini and A. Piva, "Multichannel Watermarking of color Images", Proc, IEEE Trans. Circuit Systems. Video Technology, vol. 12, no. 3, (2002), pp. 142-156.

[4] I. J. Cox, J Kilian and F. T. Leighton, "Secure spread spectrum watermarking for multimedia", IEEE Transactions on Image Processing, vol. 6, no. 12, (1997), pp. 1673-1687.

[5] Z. Wei, "Perceptual digital watermarking of images using wavelet transform", IEEE Transactions on Consumer Electronics, vol. 44, no. 4, (1998), pp. 1267-1272.

[6] R. Agarwal, M. S. Santhanam and K. Venugopalan, "Multichannel Digital Watermarking of color images using SVD", International Conference on Image Information Processing(ICIIP), (2011).

[7] WuShaSha, "Research on Digital Watermarking Technology Based on Two-Dimensional Barcode of PDF417", Nanjing University of Aeronautics and Astronautics, The Graduate School, College of Automation Engineering, (2010) December.

[8] S. Bing and G. Mei Feng, "Research on Digital Watermarking Algorithm Based on QR Barcode", JISUANJI YU XIANDAIHUA, no. 1, (2011), pp. 74-77.

[9] W. J. Van Gils, "Method of identifying objects provided with a code field containing a dot code, a device for identifying such a dot code, and a product provided with such a dot code", US Patent Number: 4745269, (1988), May 17.

\section{Authors}

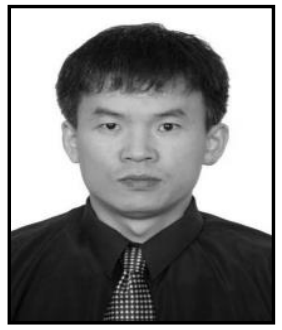

Jizhe Cui is a professor of Dept. of Information Management and Systems at Yanbian University in China. He received the Ph.D. degree from Sangmyung University, major in Computer Science in 2007. His research interests are digital watermarking, copyright management, cryptology, and computer graphics.

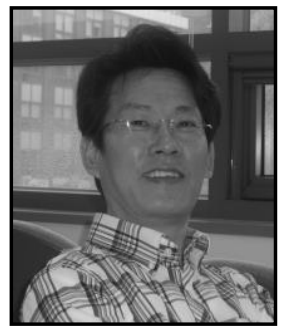

Jongweon Kim received the Ph.D. degree from University of Seoul, major in signal processing in 1995. He is currently a Professor of Dept. of Intellectual Property at Sangmyung University in Korea. He has a lot of practical experiences in the digital signal processing and copyright protection technology at the institute, the industry, and College. His research interests are in the areas of copyright protection technology, digital rights management, digital watermarking, and digital forensic marking. 